\title{
Teaching Mathematical Modeling: Art or Science?
}

\author{
Wolfgang Wiechert \\ University of Siegen, FOMAAS, \\ Paul-Bonatz-Str. 9-11, D-57068 Siegen, Germany. \\ wiechert@simtec.mb.uni-siegen.de, \\ http://www.simtec.mb.uni-siegen.de
}

\begin{abstract}
Modeling and simulation skills are two core competencies of computational science and thus should be a central part of any curriculum. While there is a well-founded theory of simulation algorithms today the teaching of modeling skills bears some intrinsic problems. The reason is that modeling is still partly an art and partly a science. As an important consequence for university education the didactic concepts for teaching modeling must be quite different from those for teaching simulation algorithms. Some experiences made with a two term course on 'Modeling and Simulation' at the University of Siegen are summarized.
\end{abstract}

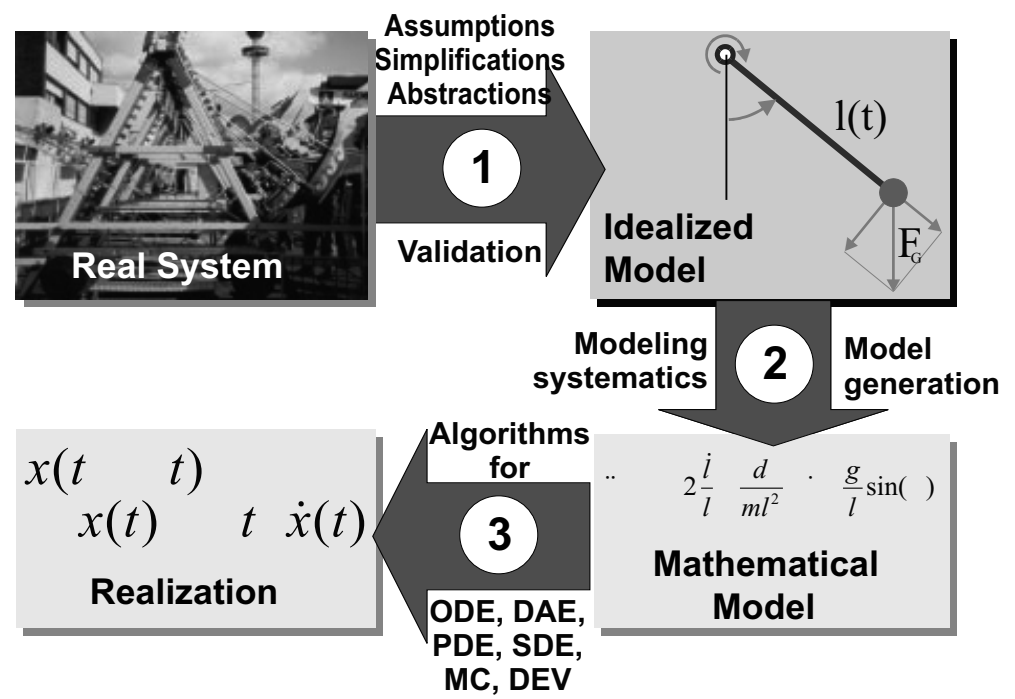

Fig. 1. General steps in modeling and simulation. The example simulation project is concerned with the modeling and simulation of a boat swing. The goal is to perform a $360^{\circ}$ looping. Details are given in the main text. 


\section{General Modeling and Simulation Methodology}

As a widely accepted approach to the modeling and simulation of a real system the overall task is divided into the following three steps, although these steps are not always clearly separated in practice (cf. Figure 1):

Step 1: From reality to an idealized model. In the first step (frequently also called 'physical modeling' [1]) the real system is replaced by an idealized model. This model is characterized by a well-defined semantics which means that once the model has been constructed there is nothing more to discuss about the physical laws that have to be used in order to describe the system. From a didactic viewpoint there are two different aspects to consider:

1. General problem of modeling reality and possible pitfalls of modeling.

2. Concepts, methods and tools for model validation.

Step 2: From the idealized model to a mathematical model. By a mathematical model a formal representation using mathematical concepts like differential equations, probability distributions or state machines is meant. The (possibly automatic) generation of the mathematical model from the idealized model essentially is an algorithmic task and is very well understood in many application disciplines (like multibody systems or electrical circuits). The following aspects are relevant in education:

1. General systematics of modeling.

2. Types of mathematical models (ODE, DAE, PDE, DES etc.).

3. Algorithms for automatic model generation.

Step 3: From the mathematical model to a computer realization. In general mathematical models represent a system in a descriptive or implicit way. A computer realization of a mathematical model means to compute a set of (possibly time and state dependent) variables that approximatively solve the model equations. This is the field of simulation algorithms which can be roughly subdivided into:

1. Numerical algorithms for AEs, ODEs, DAEs, PDEs, etc.

2. Random number generation, stochastic algorithms, MC methods etc.

3. Algorithms for discrete event systems, automata, Petri nets, etc.

\section{Teaching Mathematical Modeling}

This contribution further concentrates on the aspect of teaching mathematical modeling. It is not easy to answer the question of how to do this in the most efficient way. In the three step scheme given above the modeling activities are spread over Steps 1 and 2 while Step 3 is best described as the 'simulation' step in a narrower sense. Basically three aspects of modeling have to be integrated into a course on computational science:

Finding the right idealization of reality: This is the most difficult part in the modeling process. It is an important point that once the idealized model has been created (Step 1) we are no more confronted with reality but with 
models. In particular any mistakes made in the early phase of model building can never be corrected later on even by the highest precision numerical algorithms. These interconnections must be made very clear to the students. Otherwise there is a high risk to get lost in the difficult but always welldefined mathematical problems of designing powerful simulation algorithms while loosing the connection to the real system and the goal of the simulation project. Unfortunately, many textbooks start with the idealized model (as e.g. the mathematical or physical pendulum) instead of reality.

Building well-structured models: Although all the basic modeling decisions are made in the first step this does not imply that the mathematical model built in Step 2 is also well-structured (i.e. easy to maintain, suited for automatic model generation, well prepared for simulation algorithms). In recent years there has been great progress in the field of modeling systematics and several highly developed modeling methodologies for different model types exist [1-4]. All these methodologies are mathematically well formalized and algorithmically specified. Thus - in contrary to the first step - there is a sound scientific platform for teaching these aspects of modeling.

Model validation: Finally it should always kept in mind that modeling is an iterative procedure guided by the attempt to validate a given model. Thus model validation always accompanies the whole process of modeling and simulation. Unfortunately, the concept of 'model validity' is not at all welldefined and leads into deep methodological problems. Some well-founded concepts for model validation have been developed in a statistical framework [5] but all these methods generally rely on even more assumptions on the real system (like e.g. a known error distribution).

In summary, building the idealized model in Step 1 is still rather an art than a science. A lot of experience and specific know how about the application domain is required. On the other hand the building and automatic generation of well-structured models from a physical model (Step 2) has a sound scientific background. Likewise most of the aspects related to simulation algorithms (Step 3) are covered in well written textbooks.

\section{Three approaches to modeling}

The big challenge of university education in modeling and simulation is how to teach the art of modeling (i.e. Step 1) in an efficient way. From the experiences gathered with the lectures on 'Modeling and Simulation' at the University of Siegen the general problem of modeling can be best understood by studying 'fruitful' examples and making practical experiences with simple modeling problems. The main difficulty herein is to avoid an overload of technical problems that are more related to simulation than to modeling. Three types of examples can be distinguished. The first one is best suited for lectures, the second one for practical exercises and the last one for a seminar:

Extensive example study: The lecture starts with a simple yet not oversimplified example of a typical simulation project. It illustrates all steps of 
the modeling and simulation process without requiring any prior simulation knowledge from the students. The goal is to design a swing boat which can perform a $360^{\circ}$ looping (Figure 1). To this end the right dimensions of the swing boat must be determined, a strategy to accelerate the swing must be found and the resulting forces on the person must be computed [6]. Essentially this ends up with the classical mathematical pendulum with time dependent length. However, a lot of simplifying assumptions must be made for this reduction and some of these assumptions (e.g. on friction in the bearing) can be validated experimentally. This study serves well to give a first impression of the general relation between modeling and simulation activities in a realistic project.

Prototypical examples for certain modeling problems: These are strongly reduced examples which cover only a certain modeling problem (like e.g. friction or contacts in mechanical systems). The examples are particularly well chosen if they seduce the students to make a typical mistake. Such examples are treated for example in the first 7 units of the simulation laboratory at the University of Siegen [6] or in the EUROSIM comparisons [7]. Another source of fruitful examples turned out to be the modeling and simulation of physical toys like the woodpecker toy that illustrates the stick slip effect [8]. This is also a typical problem for the last exercise (unit 8) of the simulation laboratory where a small project has to be managed in about three days with a final presentation.

Multiscale studies of one specific system: The choice of model type usually depends on the time or space scale on which reality has to be understood. Examples by which several different scales and hence different modeling approaches can be studied are mechanical springs (Hook's law, non-linear springs, valve springs with contacts, strain inside a spring, fracture mechanics, molecular basis of elasticity), chemical reaction systems (reaction kinetics, stochastic particle system, molecular dynamics, Schrödinger equations) or manufacturing systems (strategic simulation, continuous material flow, discrete material flow, machine modeling, virtual reality).

\section{Conclusions}

Computational science involves skills from different scientific disciplines (numerics, statistics, computer science,...) and a profound knowledge of the application domain (physics, chemistry, biology, engineering,...). At the University of Siegen a general two term graduate lecture on 'Modeling and Simulation' serves as a link between the mathematical disciplines and the application domains. It demonstrates the different steps in the modeling and simulation process and clarifies the role that each single discipline plays in the whole concert. The interdisciplinary lectures are suited for engineers, mathematicians, computer scientists and natural scientists.

Due to its broad scope the mathematical demand of the introductory lecture is rather low and only the basic ideas of simulation algorithms are explained. 
Those students who want to get a deeper insight into a certain topic can attend other lectures on numerics, finite elements, computational fluid dynamics, parallel computing, software engineering etc. which are given in different departments of the University of Siegen. Thus without currently having established a dedicated masters course on computational science it is already possible for students of different disciplines to put their focus on it.

An organizational frame for computational science at the University of Siegen is given by the Research Centre for Multidisciplinary Analysis and Applied Systems Optimization (FOMAAS) which is an interdisciplinary union of scientists from the engineering, mathematics, and computer science departments [9]. FOMAAS works on the analysis, simulation and optimization of complex systems. Moreover the author is responsible for the coordination of educational activities in the German/Austrian/Swiss simulation organization ASIM. More details about the simulation courses at the University of Siegen and curricular aspects can be taken from $[6,10,11]$.

\section{References}

1. Tiller, M.: Introduction to physical modeling with Modelica, Kluwer Academic, 2001

2. Cellier, F.E.: Continuous System Modeling, Springer (1991).

3. Farlow, S.J.: Partial Differential Equations for Scientists and Engineers, Wiley (1993).

4. Fishman, G.S.: Discrete-Event Simulation: Modeling, Programming and Analysis, Springer (2001).

5. Burnham, K.P., Anderson, D.R.: Model Selection and Inference - A Practical Information-Theoretic Approach, Springer (1998).

6. Wiechert, W.: Lectures on Simulation: Lecture Notes and Materials, http://www.simtec.mb.uni-siegen.de

7. ARGESIM, Arbeitsgemeinschaft Simulation, EUROSIM Comparisons, TU Wien. http://www.argesim.org.

8. Ucke, C.: Literature data base on physical toys, http://fluorine.e20.physik.tumuenchen.de/ cucke/

9. FOMAAS (Research Centre for Multidisciplinary Analysis and Applied System Optimization): University of Siegen, http://www.fomaas.uni-siegen.de.

10. Wiechert, W.: Multimediale Lehre im Fach Simulationstechnik. Pp. 39-53 in: Großmann, K.; Wiemer, H., SIM2000 - Simulation im Maschinenbau, scan-factory, Dresden (2000).

11. Wiechert, W.: Eine Checkliste für den Aufbau einer Simulationstechnik Vorlesung. Pp. 151-156 in: Panreck, K.; Dörrscheid, F. 15. Symposium Simulationstechnik, Paderborn, SCS Publishing House (2001). 